Frédérique Liégeois, $\mathrm{PhD}$ Jacques-Donald Tournier, $\mathrm{PhD}$

Lauren Pigdon, BA Alan Connelly, $\mathrm{PhD}$ Angela T. Morgan, $\mathrm{PhD}$

Correspondence to Dr. Liégeois:

F.Liegeois@ucl.ac.uk
Supplemental data at www.neurology.org

\section{Corticobulbar tract changes as predictors of dysarthria in childhood brain injury OPEN}

\section{ABSTRACT}

Objectives: To identify corticobulbar tract changes that may predict chronic dysarthria in young people who have sustained a traumatic brain injury (TBI) in childhood using diffusion MRI tractography.

Methods: We collected diffusion-weighted MRI data from 49 participants. We compared 17 young people (mean age 17 years, 10 months; on average 8 years postinjury) with chronic dysarthria who sustained a TBI in childhood (range 3-16 years) with 2 control groups matched for age and sex: 1 group of young people who sustained a traumatic injury but had no subsequent dysarthria ( $n=15)$, and 1 group of typically developing individuals $(n=17)$. We performed tractography from spherical seed regions within the precentral gyrus white matter to track: 1) the hand-related corticospinal tract; 2) the dorsal corticobulbar tract, thought to correspond to the lips/larynx motor representation; and 3) the ventral corticobulbar tract, corresponding to the tongue representation.

Results: Despite widespread white matter damage, radial (perpendicular) diffusivity within the left dorsal corticobulbar tract was the best predictor of the presence of dysarthria after TBI. Diffusion metrics in this tract also predicted speech and oromotor performance across the whole group of TBI participants, with additional significant contributions from ventral speech tract volume in the right hemisphere.

Conclusion: An intact left dorsal corticobulbar tract seems crucial to the normal execution of speech long term after acquired injury. Examining the speech-related motor pathways using diffusion-weighted MRI tractography offers a promising prognostic tool for people with acquired, developmental, or degenerative neurologic conditions likely to affect speech. Neurology ${ }^{\oplus}$ 2013;80:926-932

\section{GLOSSARY}

$\mathbf{D W I}=$ diffusion-weighted imaging; $\mathbf{F A}=$ fractional anisotropy $\mathbf{M A N C O V A}=$ multivariate analysis of covariance; $\mathbf{R D}=$ radial diffusivity; $\mathbf{S C}=$ speech characteristics; $\mathbf{T B I}=$ traumatic brain injury; TD = typically developing; VMPAC = Verbal Motor Production Assessment for Children.

Recent advances in diffusion-weighted MRI analyses now allow the in vivo reconstruction of white matter pathways in the human brain using tractography. ${ }^{1}$ Although speech is vulnerable to numerous neurologic conditions, the association between speech disorders and integrity of the corticobulbar tracts is rarely examined.

Traumatic brain injury (TBI) is a major cause of death and disability in children ${ }^{2,3}$ and can have devastating consequences on a wide range of cognitive domains. ${ }^{4}$ Focal as well as diffuse brain changes have been observed after TBI, including long-term white matter alterations $s^{5,6}$ that can be now be revealed using diffusion-weighted imaging (DWI). ${ }^{7-11}$ Among children with moderate and severe TBI, approximately $20 \%$ may have chronic dysarthria, ${ }^{12}$ which can affect speech across articulation, respiration, phonation, prosody, and resonance. Given the potential for functional reorganization seen in the immature brain, ${ }^{6,13,14}$ studies in the chronic stage are crucial to unravel the neural basis of those deficits that do persist long term after TBI in childhood. Because a recent review indicated that damage along the corticobulbar/corticospinal tracts was a common feature in children with dysarthria, ${ }^{15}$ we used tractography to test the

\footnotetext{
From the Developmental Cognitive Neuroscience Unit (F.L.), UCL Institute of Child Health, London, UK; Florey Institute of Neuroscience and Mental Health (J.-D.T., A.C., A.T.M.), Melbourne; and Murdoch Childrens Research Institute (L.P., A.T.M.), Royal Children's Hospital (A.T.M), and Department of Paediatrics (A.T.M.), University of Melbourne, Melbourne, Australia.

Go to Neurology.org for full disclosures. Funding information and disclosures deemed relevant by the authors, if any, are provided at the end of the article. This is an open access article distributed under the Creative Commons Attribution License, which permits unrestricted use, distribution, and reproduction in any medium, provided the original work is properly cited.
} 
hypothesis that chronic dysarthria after TBI is associated with damage to the corticobulbar tracts.

We recruited 3 carefully matched groups of young people: 2 after childhood TBI (1 with and 1 without subsequent dysarthria), and 1 of typically developing (TD) individuals. To help inform prognosis, we investigated which tractography-derived measures best predicted both presence of dysarthria and speech/oromotor performance long term after TBI.

METHODS Participants. We selected participants from medical charts from the Royal Children's Hospital, Melbourne, Australia, a tertiary pediatric care center with specialist acute and rehabilitation acquired brain injury teams. For each TBI participant with dysarthria (TBI+ group), we recruited 2 control participants, selected to be individually matched for sex and age ( \pm 1 or 2 years). For the group post-TBI without dysarthria (TBI-), a diagnosis of speech disorder at any stage post-TBI was an additional exclusion criterion. The TBIparticipants were also matched to TBI+ participants based on injury severity, type of injury, and handedness wherever possible (see e-Methods for recruitment details and table e-1 on the Neurology ${ }^{\circledR}$ Web site at www.neurology.org). We also recruited a control group of TD participants. The final sample included 49 participants, 17 in each of the TBI+ and TD groups, and 15 in the TBI- group. In the TBI+ group, all participants showed signs of dysarthria in the acute stage ( 8 severe, 3 moderate-severe, 3 moderate, 2 mildmoderate, and 1 unknown) within the first 2 months postinjury.

Ethics approval and patients' consent. We obtained Ethics approval from the Royal Children's Hospital Human Research Ethics Committee, approval number 27083. All participants gave informed consent and assent (if younger than 18 years). All guardians of participants gave informed consent.

Descriptive measures. Speech diagnosis. All TBI participants underwent a detailed speech assessment by an experienced speechlanguage pathologist (A.M.). As in previous studies, ${ }^{16,17}$ we characterized dysarthric speech using the Mayo Clinic classification system, which involves rating impairment across articulation (e.g., imprecise consonants and vowels), prosody (e.g., reduced rate), resonance (e.g., hypernasality), phonation (e.g., hoarse voice quality), and respiration (e.g., audible inspiration). Dysarthria severity ranged from mild ( $\mathrm{n}=11)$, mild-moderate $(\mathrm{n}=2)$, moderate $(\mathrm{n}=3)$ to severe (1 case). We also assessed speech and oromotor function using the Verbal Motor Production Assessment for Children (VMPAC ${ }^{18,19}$, see our previous study ${ }^{20}$ for assessment details), which examines neuromuscular integrity of the speech system. This test provides indices of impairment for 5 areas, but the domains of specific interest here were 1) speech characteristics (SC), which requires a rating of participants' quality of speech production across domains of resonance, articulation, pitch, and respiration, and therefore assesses several aspects of speech production that may be affected by dysarthria, and 2) focal oromotor control, which measures a participant's ability to perform oromotor and speech movements in sequence and isolation. We also assessed IQ (see e-Methods).

Clinical variables. We extracted initial injury characteristics from medical charts. Brain abnormalities viewed mostly on CT scans had been assessed by a clinical radiologist at the time of injury. TBI severity in the acute stage was mainly moderate-severe (see table e-1)
MRI acquisition. We collected MRI data on a 3-tesla Tim Trio MRI scanner (Siemens Medical Solutions, Erlangen, Germany). We also collected high-resolution T1-weighted images with an isotropic resolution of $0.9 \mathrm{~mm}$ (inversion time $=900$ milliseconds, repetition time $=1,900$ milliseconds, echo time $=2.6$ milliseconds, flip angle $=9^{\circ}$, matrix size $256 \times 256,160$ partitions) for volumetric analyses.

In addition, we acquired a single diffusion-weighted dataset consisting of 60 directions using a twice-refocused, spin-echo, echo planar imaging sequence with $\mathrm{b}$ value $=3,000 \mathrm{~s} / \mathrm{mm}^{2}$, echo time/ repetition time $=110 / 8,300$ milliseconds, field of view $=240 \times$ $240 \mathrm{~mm}$, matrix size $=96 \times 96$, slice thickness $=2.5 \mathrm{~mm}$ (isotropic voxel size $=2.5 \times 2.5 \times 2.5 \mathrm{~mm}$ ), 60 contiguous axial slices (total acquisition $\approx 9$ minutes).

Preprocessing of MRI datasets. We segmented T1-weighted datasets into gray matter, white matter, and CSF using the New Segmentation toolbox implemented in SPM8 (http://www.fil.ion.ucl. ac.uk/spm/software/spm8/) and smoothed using a full-width at halfmaximum 4-mm Gaussian kernel. We extracted global white matter volumes (in voxels) using the Easyvolume tool (developed by C. Pernet; http://www.sbirc.ed.ac.uk/ld//LCL_M1.html) implemented in SPM8.

We processed DWI datasets using the MRtrix package ${ }^{21}$ (J.-D. Tournier, Brain Research Institute, Melbourne, Australia, http://www.brain.org.au/software/). We extracted fractional anisotropy (FA) and eigenvector maps. We then used constrained spherical deconvolution to estimate the distribution of fiber orientations. ${ }^{22,23}$ This method is advantageous relative to the diffusion tensor model in areas with multiple crossing fibers. ${ }^{21,24}$ Given the possible differences in gross anatomy among individuals after TBI and the presence of focal lesions in some, tractography analyses were performed in each participant's native space. Global white matter volume was used as a covariate in the subsequent track volume analyses.

Tractography. A single rater (F.L.), blinded to participants' speech status, performed tractography of the motor tracts using the MRtrix software package. ${ }^{21}$ Fiber orientations were estimated using constrained spherical deconvolution. ${ }^{23}$ We performed tracking (using a probabilistic streamlines algorithm ${ }^{21}$ ) from spherical seed regions (7-mm radius) located at 3 axial levels of the precentral gyrus white matter between the hand representation (hand omega) and the most ventral part of the precentral gyrus. The 2 regions ventral to the hand representation are reported to correspond to the lips/larynx and tongue representations in the motor cortex (termed "face" and "tongue," respectively, in the study by Pan et al., ${ }^{25}$ 2012). A pons region of interest was delineated from the axial slice where the corticobulbar/corticospinal tract lies, between the transverse pontine fibers and the middle cerebellar peduncle, and was used as inclusion region (see e-Methods and figure e- 1 for details). We set the maximum number of streamlines generated at 10,000 and retained a maximum of 1,000 streamlines passing through both the seed and inclusion regions. We extracted mean FA and mean radial diffusivity (RD) from all 3 tracks within each hemisphere using MRtrix.

Statistical analysis of MRI-derived data. We analyzed all individual numerical data using the statistical package SPSS 16.0 for Windows (SPSS Inc., Chicago, IL). To reduce the risk of type I error, we entered track-derived measures into multivariate analyses of covariance (MANCOVAs), with age and total white matter volumes as covariates. We did not use track volume as a covariate (see e-Methods).

We explored significant effects further using univariate analyses. The $\alpha$ level was set at 0.05 (2-tailed) and adjusted (Bonferroni) to correct for multiple comparisons when performing post hoc analyses. Nonparametric methods (Mann-Whitney tests) were used for confirmation when appropriate. 
We identified MRI predictors of the presence of dysarthria after TBI using binary logistic regressions. We examined MRI predictors of speech performance (VMPAC subtest scores) using exploratory linear regressions. Given the high negative correlation between $\mathrm{RD}$ and FA, and the relationship between RD/FA and volume, we examined these in separate regressions. We examined multicollinearity (calculated using the tolerance index and variance inflation factor) and linearity of the logit to ensure no assumptions were violated.

RESULTS Sample characteristics. The 3 groups were adequately matched for demographic characteristics (table 1). In addition, the 2 groups post-TBI did not differ on any injury-related measures. There was only a trend for differences in intelligence, with all scores within the normal range. The group with dysarthria scored lower than both comparison groups on both subtests of the VMPAC (table 1). There was also a trend for reduced global white matter in the TBI+ group relative to the TD group.

Tractography. Tractography successfully delineated all tracts in all participants (see figure 1 for illustrative examples), apart from 8 instances in which no streamlines were generated. In those cases, we treated tractography-derived measures as missing values. We included the other 286 track values in the analyses.

Tractography analyses. Track volumes. Track volumes differed only slightly between groups (trend in multivariate analyses: $F=1.78, p=0.067)$ with differences in the left hand-related track only $(F=4.69, p=0.014)$, because of a reduction in the $\mathrm{TBI}+$ group relative to the TD group (Bonferroni-corrected $p=0.012$ ) (figure e-2). No other group differences were found.

Mean $F A$. MANCOVA revealed an effect of group $(F=2.03, p=0.035)$, with significant effects in the left hand-related tracks bilaterally and in the left dorsal speech track (table 2 and figure 2, top row). The TBI+ group had reduced FA relative to TD peers bilaterally in the hand-related tracks. Relative to the TBI- group, FA was reduced in the right hand-related and left dorsal speech tracks. The TBI- group showed no differences relative to the TD group.

Mean $R \boldsymbol{D}$. MANCOVA revealed a borderline effect of group $(F=1.61, p=0.081)$. The TBI+ group had higher RD than TD peers in both hand-related tracks, as well as in the left dorsal speech track (trend). In addition, the TBI+ participants had higher RD than TBI- peers in the left dorsal speech track (table 3 and figure 2, bottom row). Overall, these results are consistent with the FA findings. The TBI- group showed no differences relative to the TD group.

Additional analyses of covariance (age and global white matter as covariates) with group as between-subject factor and hemisphere as a within-subject factor revealed a group by hemisphere interaction for FA only, and only in the dorsal speech track $(F=3.75, p=0.032)$.

Predictors of presence of dysarthria within the TBI group. We conducted 2 stepwise logistic regressions (1 for FA, 1 for $\mathrm{RD}$ ) using DWI-derived measures from the 6 tracks as predictors. A test of the full model against a constant-only model revealed that $\mathrm{RD}$ and FA within the left dorsal speech track were both the best and unique predictors of dysarthria post-TBI, consistent with analysis of covariance results described earlier. Both models were statistically significant $\left(\chi^{2}=6.72, p=0.01\right.$ for FA; $\chi^{2}=8.28, p<0.005$ for $\mathrm{RD}$ ). The best model, with $\mathrm{RD}$ as predictor, correctly classified $81 \%$ of the sample (sensitivity $83 \%$, specificity $79 \%$; $-2 \log$ likelihood $=27.6$; Wald statistic $=5.64, p=0.018$; Nagelkerke $R^{2}=0.36$; Hosmer-Lemeshow goodnessof-fit test statistic $=0.48)$. When $\mathrm{RD}$ is increased by 1 unit $\left(1 \times 10^{-4} \mathrm{~mm}^{2} / \mathrm{s}\right)$ in the left dorsal speech track, patients with TBI are 8 more times likely to have dysarthria.

Table 1 Sample demographics and injury characteristics

\begin{tabular}{|c|c|c|c|c|c|c|c|}
\hline & \multicolumn{3}{|l|}{ Group } & \multirow[b]{2}{*}{ Group effect } & \multicolumn{3}{|c|}{ Group comparisons $^{a}$} \\
\hline & $\mathrm{TBI}+(\mathrm{n}=17)$ & $\mathrm{TBI}-(\mathrm{n}=15)$ & $\operatorname{TD}(n=17)$ & & TBI+ vs TD & TBI- vs TD & TBI+ vs TBI- \\
\hline Mean age: y; mo (SD) & $17 ; 10(4 ; 0)$ & $18 ; 8(4 ; 3)$ & $18 ; 3(4 ; 10)$ & NS & - & - & - \\
\hline Sex, F:M & $9: 8$ & $9: 6$ & $9: 8$ & NS & - & - & - \\
\hline Severity of injury ${ }^{b}$ : mild, moderate-severe & 1,16 & 1,14 & - & - & - & - & NS \\
\hline Mean age at injury: $y ;$ mo (range) & $9 ; 4(3-16)$ & $10 ; 10(4-16)$ & - & - & - & - & NS \\
\hline Type of injury: MVA, fall, blunt trauma & $10,6,1$ & $11,3,1$ & - & - & - & - & NS \\
\hline Mean time since injury: y; mo (SD) & $8(4 ; 10)$ & $7 ; 9(2 ; 1)$ & - & - & - & - & NS \\
\hline Mean IQ (SD) & $90.7(12)$ & $99.6(13)$ & 103.7 (13) & $F=4.80, p=0.013$ & $p=0.012$ & NS & NS \\
\hline Mean FOC score (SD) & 84.1 (9.2) & $95(4.0)$ & $96.5(4.3)$ & $F=19.06, p<0.001$ & $p<0.001$ & NS & $p<0.001$ \\
\hline Mean SC score (SD) & $73.0(26.7)$ & $96.1(10.2)$ & $98.3(7.0)$ & $F=10.78, p<0.001$ & $p<0.001$ & NS & $p=0.002$ \\
\hline
\end{tabular}

Abbreviations: FOC = focal oromotor control; MVA = motor vehicle accident; NS = not significant ( $p>0.10)$; SC $=$ speech characteristics;

$\mathrm{TBI}+$ = post-traumatic brain injury with dysarthria; TBI- = post-traumatic brain injury without dysarthria; TD = typically developing

a Post hoc Bonferroni-corrected values.

${ }^{\mathrm{b}}$ As assessed using the Mayo Clinic TBI classification system. 


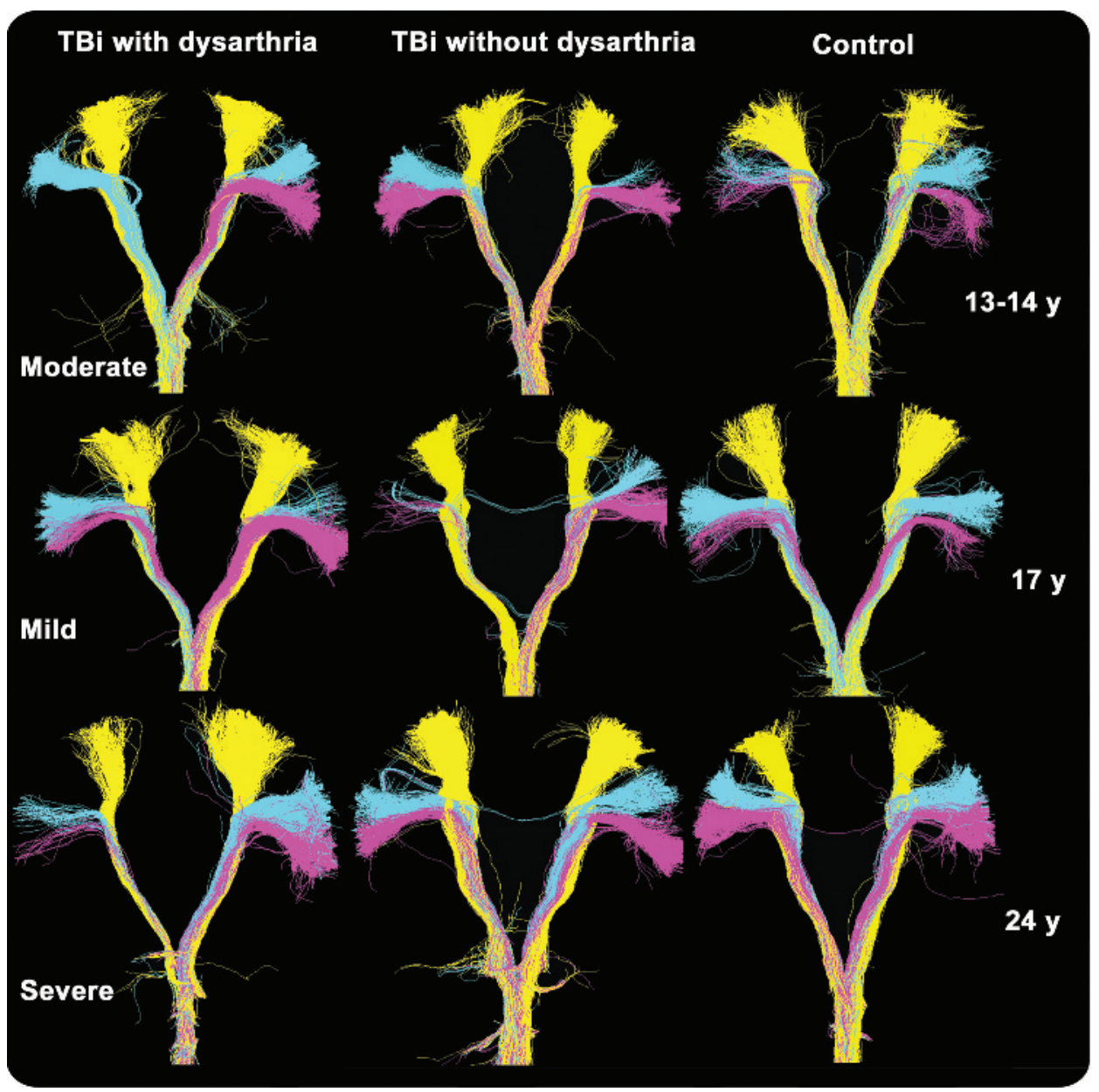

Coronal projections of hand-related (yellow), dorsal speech (blue), and ventral speech (pink) tracks for 9 randomly selected participants across the age range, and (for the traumatic brain injury [TBI]+ participants) across dysarthria severity. Note the variability of track thickness and volume irrespective of group. Left hemisphere is on the right. The age of the 3 participants in each row is indicated on the right.

Predictors of speech performance in the whole TBI sample. Speech characteristics. Three stepwise linear regression analyses using metrics (FA, RD, and volume) from the 4 speech tracks as independent variables revealed 3 significant models, the first with FA within the left dorsal speech track as predictor $\left(R_{\text {adjusted }}^{2}=0.12\right.$, $F=4.47, p=0.045)$, the second with $\mathrm{RD}$ within the left dorsal speech track as a unique predictor $\left(R_{\text {adjusted }}^{2}=0.17, F=6.30, p=0.02\right)$, and the third with volume of the right ventral speech track as a unique predictor $\left(R_{\text {adjusted }}^{2}=0.19, F=8.30, p=0.007\right)$.

Because these 2 latter measures were not correlated, they were entered into a final regression. This final model was significant and explained a higher proportion of the variance in SC scores $\left(R_{\text {adjusted }}^{2}=\right.$ $0.32, F=8.38, p=0.001)$.

Focal oromotor control. Stepwise linear regressions using the same independent variables as above again showed 3 significant models, the first with RD within the left dorsal speech track as predictor $\left(R_{\text {adjusted }}^{2}=0.20\right.$,
$F=7.34, p=0.012)$, the second with FA within the same track $\left(R_{\text {adjusted }}^{2}=0.15, F=5.54, p=0.027\right)$, the third with volume of the right ventral and left dorsal speech track $\left(R_{\text {adjusted }}^{2}=0.29, F=7.21, p=0.003\right)$. Because volume of right ventral track and $\mathrm{RD}$ of the left dorsal track were not correlated, they were entered into a final regression. This final model was significant and explained a higher proportion of the variance in SC scores $\left(R_{\text {adjusted }}^{2}=0.30, F=7.61, p=0.002\right)$.

Although these results have to be interpreted with caution given the modest sample size and small spread of scores, they converge to indicate that diffusion metrics from the left dorsal speech track have an association with 1) the presence of dysarthria, and 2) the degree of speech and oromotor impairment after TBI. Volumetric measures from the contralateral ventral speech track, however, also contributed to the latter variance, with higher volumes associated with better performance.

Effect of age at injury on diffusion metrics. In the TBIgroup, FA in the left hand-related track increased 


\begin{tabular}{|c|c|c|c|c|}
\hline \multicolumn{5}{|c|}{$\begin{array}{l}\text { Group differences in mean fractional anisotropy as assessed by } \\
\text { analyses of covariance (age as covariate) for each track }\end{array}$} \\
\hline Track & $F$ & $p$ Value & Group comparisons & p Value ${ }^{b}$ \\
\hline \multirow[t]{2}{*}{ Left hand-related ${ }^{c}$} & 6.50 & 0.003 & $\mathrm{TD}>\mathrm{TBI}+$ & $0.003^{d}$ \\
\hline & & & TBI- > TBI+ & $0.07^{d}$ \\
\hline \multirow[t]{2}{*}{ Right hand-related ${ }^{c}$} & 4.14 & 0.023 & $\mathrm{TD}>\mathrm{TBI}+$ & $0.04^{d}$ \\
\hline & & & $\mathrm{TBI}->\mathrm{TBI}+$ & $0.08^{d}$ \\
\hline \multirow[t]{2}{*}{ Left dorsal speech } & 4.25 & 0.020 & $\mathrm{TD}>\mathrm{TBI}+$ & NS \\
\hline & & & $\mathrm{TBI}->\mathrm{TBI}+$ & 0.02 \\
\hline \multirow[t]{2}{*}{ Right dorsal speech } & 0.20 & NS & $\mathrm{TD}>\mathrm{TBI}+$ & NS \\
\hline & & & $\mathrm{TBI}->\mathrm{TBI}+$ & NS \\
\hline \multirow[t]{2}{*}{ Left ventral speech } & 1.98 & NS & $\mathrm{TD}>\mathrm{TBI}+$ & NS \\
\hline & & & $\mathrm{TBI}->\mathrm{TBI}+$ & NS \\
\hline \multirow[t]{2}{*}{ Right ventral speech } & 0.47 & NS & $\mathrm{TBI}+>\mathrm{TD}$ & NS \\
\hline & & & TBI- > TBI+ & NS \\
\hline
\end{tabular}

Abbreviations: NS = not significant $(p>0.10) ; \mathrm{TBI}+=$ post-traumatic brain injury with dysarthria; TBI - = post-traumatic brain injury without dysarthria; TD = typically developing.

${ }^{a}$ Note that only statistically significant $(p<0.05)$ or borderline $(0.05<p<0.10)$ effects are reported.

${ }^{\mathrm{b}}$ Bonferroni corrected.

${ }^{c}$ Variable violated equality of variance error assumption.

d Significant difference using nonparametric test (Mann-Whitney).

with age at injury $(r=0.57, p=0.027)$, whereas RD decreased marginally $(r=-0.49, p=0.063)$. In contrast, in the TBI+ group, $\mathrm{RD}$ increased with age at injury in both the left hand-related $(r=0.67$, $p=0.003)$ and in the left dorsal speech track $(r=$ $0.50, p=0.04)$, whereas FA decreased in the left hand-related track only $(r=-0.64, p=0.006)$. Relationships between age and diffusion metrics are reported in e-Results.

DISCUSSION Our tractography approach revealed that diffusion metrics from the left dorsal speech track were the best predictors of both presence of dysarthria and speech and oromotor outcome. This study provides converging evidence suggesting that the left dorsal corticobulbar tract is crucial to the normal execution of speech long term after brain injury acquired in childhood.

Our findings suggest that the changes reflected in diffusion parameters in the left corticobulbar tract may be at the root of acquired dysarthria, beyond global white matter reductions after TBI. These results mirror those found for hand movements after TBI, where FA reductions in the corticospinal tract are associated with motor ${ }^{26,27}$ or visuomotor ${ }^{28}$ impairment. Our findings are also consistent with the few reports of acquired dysarthria in children who have had infarcts, whereby lesions mostly encompass the left putamen/internal capsule region, ${ }^{15}$ through which this tract runs. ${ }^{25}$ Finally, the association between left hemisphere speech-related tracts and speech function after TBI is in agreement with the association between left hemisphere infarct and acute dysarthria in adults. ${ }^{29,30}$

Figure 2 Diffusion metrics within the 6 tracks for each group

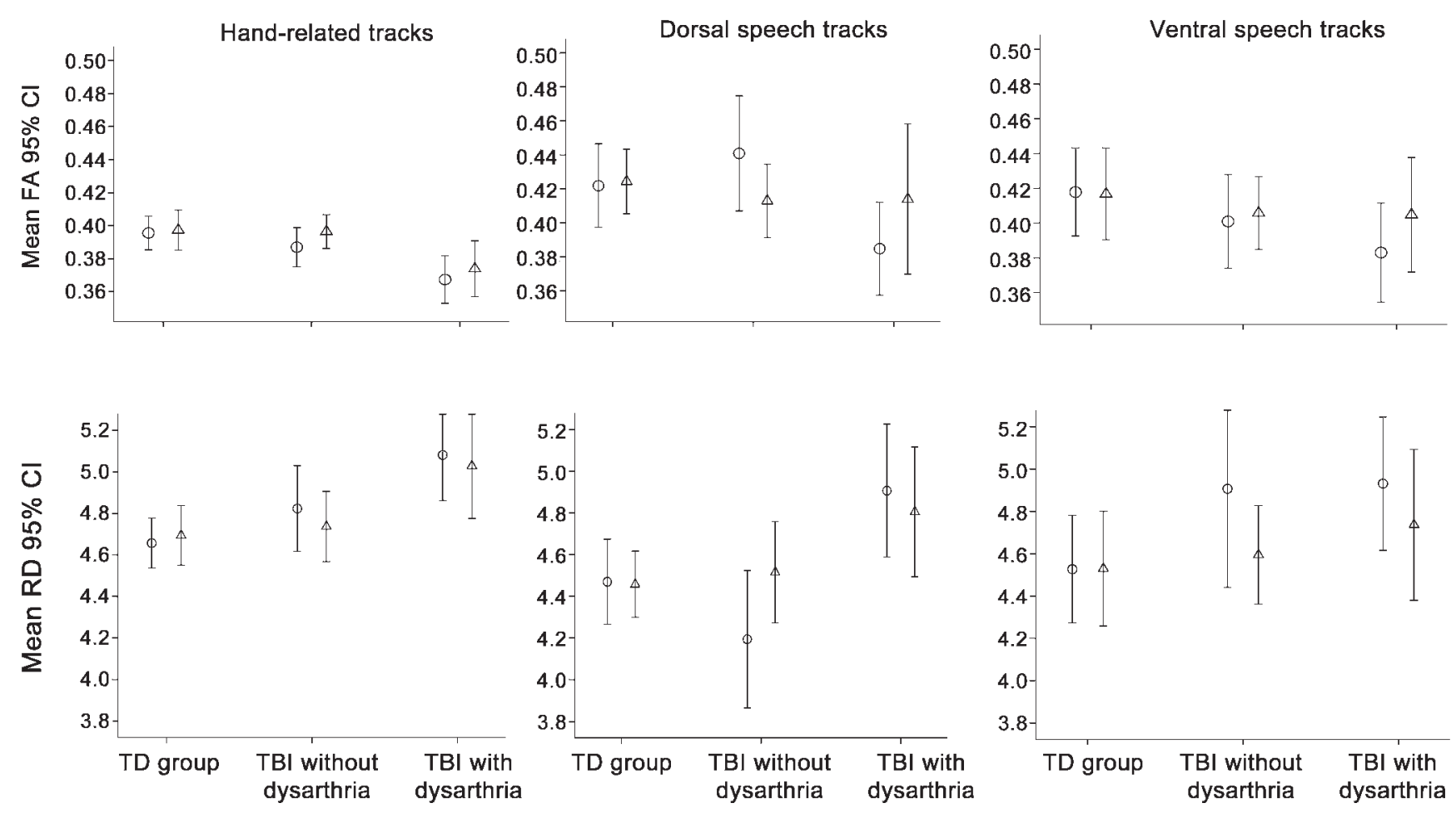

Mean track fractional anisotropy ( $F A$, top row) and radial diffusivity $\left(\mathrm{RD}, \times 10^{-4} \mathrm{~mm}^{2} / \mathrm{s}\right.$, bottom row) in each group. Circles $=$ left hemisphere; triangles $=$ right hemisphere. $\mathrm{Cl}=$ confidence interval; $\mathrm{TBI}=$ traumatic brain injury; TD = typically developing. 
Table 3 Group differences in mean radial diffusivity as assessed by analyses of covariance (age as covariate) for each track ${ }^{a}$

\begin{tabular}{|c|c|c|c|c|}
\hline Track & $F$ & $p$ Value & Group comparisons & $p$ Value $^{b}$ \\
\hline \multirow[t]{2}{*}{ Left hand-related ${ }^{c}$} & 6.63 & 0.003 & $\mathrm{TD}<\mathrm{TBI}+$ & $0.002^{d}$ \\
\hline & & & TBI $-<\mathrm{TBI}+$ & NS \\
\hline \multirow[t]{2}{*}{ Right hand-related ${ }^{c}$} & 3.94 & 0.027 & $\mathrm{TD}<\mathrm{TBI}+$ & 0.03 \\
\hline & & & $\mathrm{TBI}-<\mathrm{TBI}+$ & NS \\
\hline \multirow[t]{2}{*}{ Left dorsal speech } & 5.85 & 0.006 & $\mathrm{TD}<\mathrm{TBI}+$ & 0.066 \\
\hline & & & TBI $-<\mathrm{TBI}+$ & 0.006 \\
\hline \multirow[t]{2}{*}{ Right dorsal speech } & 2.80 & 0.073 & $\mathrm{TD}<\mathrm{TBI}+$ & 0.09 \\
\hline & & & $\mathrm{TBI}-<\mathrm{TBI}+$ & NS \\
\hline \multirow[t]{2}{*}{ Left ventral speech } & 1.82 & NS & $\mathrm{TD}<\mathrm{TBI}+$ & NS \\
\hline & & & $\mathrm{TBI}-<\mathrm{TBI}+$ & NS \\
\hline \multirow[t]{2}{*}{ Right ventral speech } & 0.79 & NS & $\mathrm{TD}<\mathrm{TBI}+$ & NS \\
\hline & & & TBI $-<$ TBI + & NS \\
\hline
\end{tabular}

Abbreviations: NS = not significant ( $p>0.10) ; \mathrm{TBI}+=$ post-traumatic brain injury with dysarthria; TBI- = post-traumatic brain injury without dysarthria; TD = typically developing.

${ }^{a}$ Note that only statistically significant $(p<0.05)$ or borderline $(0.05<p<0.10)$ effects are reported.

${ }^{\mathrm{b}}$ Bonferroni corrected.

${ }^{c}$ Variable violated equality of variance error assumption.

d Significant difference using nonparametric test (Mann-Whitney).

The range of impairment and speech performance in our sample was additionally related to the diffusion metrics of the right hemisphere tracks, indicating that additional right hemisphere damage results in the most severe impairments. These preliminary findings indicate that the contralateral hemisphere has an important role in functional compensation, possibly similar to the right hemisphere reorganization of language function after early brain injury. ${ }^{31}$

The seed regions used for tracking the dorsal speech pathways were located immediately ventral to the hand representation in the motor cortex. In adults, lesions encompassing this cortical region are associated with severe dysarthria, ${ }^{32,33}$ corroborating its potential crucial role for articulatory function. Importantly, the damage to the dorsal corticobulbar tract was not present at the macroscopic level, grossly measured here using track volume, because we have little evidence of frank interruption or thinning of tracks in participants with dysarthria. Instead, crucial measures were FA and RD changes, which can only be measured using diffusion MRI. It has been suggested that chronic changes in FA and $\mathrm{RD}$ indicate both axonal and myelin damage. ${ }^{34}$ Having no direct measure of microstructural changes within the speech tracts examined here, we can only speculate that the diffusion changes observed are a result of post-TBI axonal injury or degeneration. ${ }^{7}$

Age at injury is hypothesized to have an important role in outcome post-TBI, as some authors argue that normal maturation and growth may be arrested as a result of injury. ${ }^{4,35}$ This arrested development effectively results in early vulnerability, where greater long-term deficits occur in children injured at a younger age, especially if the injury is severe. ${ }^{6,14}$ Our present findings were consistent with this hypothesis in the group without dysarthria, whereby later age at injury resulted in "healthier" track metrics in the left hand-related corticospinal tract. In contrast, however, the opposite trend was found in the TBI group with dysarthria. In the latter group, injury occurring later was associated with higher $\mathrm{RD}$ in the left dorsal speech tracks, suggesting instead that later injuries are a disadvantage. In the domain of motor speech, an early plasticity theory may therefore better account for our findings. Of note, diffusion metrics in the TBI participants with dysarthria did not change with chronological age as they did in the control group, suggesting that developmental trajectories of both hand- and speech-related tracts may indeed be significantly altered, even possibly "arrested," after TBI.

This study is not without the limitations that are inherent to any DWI-based study, such as the influence of crossing fibers on diffusion metrics, the difficulty in interpreting diffusion changes, and the large number of non-independent measurements. We believe that we have minimized these issues by 1 ) using a tractography algorithm proven to be robust to crossing fibers, ${ }^{21}$ 2) recruiting 2 carefully matched groups of control participants, and 3) analyzing white matter changes both between and within groups. The retrospective nature of the study did not allow us to establish the time at which dysarthria or corticobulbar tract changes occur after injury. Our chronic findings reveal the need for a prospective longitudinal design to uncover how early white matter changes can inform prognosis, therefore enabling prompt intervention. Finally, our results will need replication in a larger sample and a wider range of dysarthria severity.

Clinically, the present findings suggest that damage to the left dorsal corticobulbar tract may be a predictive marker of subsequent dysarthria in individuals with acquired brain injury. Tractography of the left corticobulbar tract therefore offers a promising way to provide accurate brain-based prognosis of speech deficits, independent of other motor deficits, in both children and adults.

\section{AUTHOR CONTRIBUTIONS}

Dr. Liégeois, Dr. Morgan, Dr. Tournier, and Dr. Connelly designed the research and revised the manuscript. Dr. Morgan, Miss Pigdon, Dr. Connelly, and Dr. Tournier performed the research. Dr. Liégeois and Dr. Morgan analyzed and interpreted the data.

\section{ACKNOWLEDGMENT}

The authors thank all participants and their families for their time and support. The authors also thank Shawna Farquharson and her team of radiographers for scanning participants. J.-D.T. and A.C. are grateful to the Operational Infrastructure Support Program of the State Government of Victoria for their support.

\section{STUDY FUNDING}

This study was supported by grant D131 from Victorian Neurotrauma Initiative, Australia (now Transport Accident Commission, TAC), 
awarded to A.T.M., F.L., A.C., and J.-D.T.; and National Health and Medical Research Council grant 607315 awarded to A.T.M.

\section{DISCLOSURE}

The authors report no disclosures relevant to the manuscript. Go to Neurology.org for full disclosures.

Received August 3, 2012. Accepted in final form November 9, 2012.

\section{REFERENCES}

1. Lawes INC, Barrick TR, Murugam V, et al. Atlas-based segmentation of white matter tracts of the human brain using diffusion tensor tractography and comparison with classical dissection. Neuroimage 2008;39:62-79.

2. Keenan HT, Bratton SL. Epidemiology and outcomes of pediatric traumatic brain injury. Dev Neurosci 2006;28:256-263.

3. Parslow RC, Morris KP, Tasker RC, Forsyth RJ, Hawley CA Epidemiology of traumatic brain injury in children receiving intensive care in the UK. Arch Dis Child 2005;90:1182-1187.

4. Anderson V, Brown S, Newitt H, Hoile H. Long-term outcome from childhood traumatic brain injury: intellectual ability, personality, and quality of life. Neuropsychology 2011;25:176-184.

5. Tasker RC. Changes in white matter late after severe traumatic brain injury in childhood. Dev Neurosci 2006;28:302-308.

6. Levin HS. Neuroplasticity following non-penetrating traumatic brain injury. Brain Inj 2003;17:665-674.

7. Wang JY, Bakhadirov $\mathrm{K}$, Abdi $\mathrm{H}$, et al. Longitudinal changes of structural connectivity in traumatic axonal injury. Neurology 2011;77:818-826.

8. Oni MB, Wilde EA, Bigler ED, et al. Diffusion tensor imaging analysis of frontal lobes in pediatric traumatic brain injury. J Child Neurol 2010;25:976-984

9. Yallampalli R, Wilde EA, Bigler ED, et al. Acute white matter differences in the fornix following mild traumatic brain injury using diffusion tensor imaging. J Neuroimaging Epub 2010 Nov 17.

10. Suskauer SJ, Huisman TA. Neuroimaging in pediatric traumatic brain injury: current and future predictors of functiona outcome. Dev Disabil Res Rev 2009;15:117-123.

11. Singh M, Jeong J, Hwang D, Sungkarat W, Gruen P. Novel DTI methodology to detect and quantify injured regions and affected brain pathways in traumatic brain injury. Magn Reson Imaging 2010;28:22-40.

12. Morgan AT, Mageandran SD, Mei C. Incidence and clinical presentation of dysarthria and dysphagia in the acute setting following pediatric traumatic brain injury. Child Care Health Dev 2010;36:44-53.

13. Anderson V, Spencer-Smith M, Wood A. Do children really recover better? Neurobehavioural plasticity after early brain insult. Brain 2011;134(pt 8):2197-2221.

14. Anderson V, Catroppa C, Morse S, Haritou F, Rosenfeld J. Functional plasticity or vulnerability after early brain injury? Pediatrics 2005;116:1374-1382.

15. Liégeois FJ, Morgan AT. Neural bases of childhood speech disorders: lateralization and plasticity for speech functions during development. Neurosci Biobehav Rev 2012;36:439-458.

16. Liégeois F, Morgan AT, Stewart LH, Helen Cross J, Vogel AP, Vargha-Khadem F. Speech and oral motor profile after childhood hemispherectomy. Brain Lang 2010;114:126-134.

17. Morgan AT, Liégeois F, Liederkerke C, et al. Role of cerebellum in fine speech control in childhood: persistent dysarthria after surgical treatment for posterior fossa tumour. Brain Lang 2011;117:69-76.
18. Hayden D, Square P. Verbal Motor Production Assessment for Children (VMPAC). Oxford: Harcourt Assessment; 1999.

19. McCauley RJ, Strand EA. A review of standardized tests of nonverbal oral and speech motor performance in children. Am J Speech Lang Pathol 2008;17:81-91.

20. Northam GB, Liégeois F, Chong WK, et al. Speech and oromotor outcome in adolescents born preterm: relationship to motor tract integrity. J Pediatr 2012;160:402-408.

21. Tournier JD, Calamante F, Connelly A. MRtrix: diffusion tractography in crossing fiber regions. Int J Imaging Syst Technol 2012;22:53-66.

22. Tournier JD, Calamante F, Gadian DG, Connelly A. Direct estimation of the fiber orientation density function from diffusion-weighted MRI data using spherical deconvolution. Neuroimage 2004;23:1176-1185.

23. Tournier JD, Calamante F, Connelly A. Robust determination of the fibre orientation distribution in diffusion MRI: non-negativity constrained super-resolved spherical deconvolution. Neuroimage 2007;35:1459-1472.

24. Tournier JD, Yeh CH, Calamante F, Cho KH, Connelly A, Lin CP. Resolving crossing fibres using constrained spherical deconvolution: validation using diffusion-weighted imaging phantom data. Neuroimage 2008;42:617-625.

25. Pan C, Peck KK, Young RJ, Holodny AI. Somatotopic organization of motor pathways in the internal capsule: a probabilistic diffusion tractography study. AJNR Am J Neuroradiol 2012;33:1274-1280.

26. Caeyenberghs K, Leemans A, Geurts M, et al. Correlations between white matter integrity and motor function in traumatic brain injury patients. Neurorehabil Neural Repair 2011;25:492-502.

27. Shin SS, Verstynen T, Pathak S, et al. High-definition fiber tracking for assessment of neurological deficit in a case of traumatic brain injury: finding, visualizing, and interpreting small sites of damage. J Neurosurg 2012;116:1062-1069.

28. Caeyenberghs K, Leemans A, Geurts M, et al. Brainbehavior relationships in young traumatic brain injury patients: fractional anisotropy measures are highly correlated with dynamic visuomotor tracking performance. Neuropsychologia 2010;48:1472-1482.

29. Urban PP, Rolke R, Wicht $S$, et al. Left-hemispheric dominance for articulation: a prospective study on acute ischaemic dysarthria at different localizations. Brain 2006;129(pt 3):767-777.

30. Urban PP, Hopf HC, Fleischer S, Zorowka PG, MüllerForell W. Impaired cortico-bulbar tract function in dysarthria due to hemispheric stroke: functional testing using transcranial magnetic stimulation. Brain 1997;120(pt 6):1077-1084.

31. Liégeois F, Connelly A, Cross JH, et al. Language reorganization in children with early-onset lesions of the left hemisphere: an fMRI study. Brain 2004;127(pt 6):1229-1236.

32. Terao Y, Ugawa Y, Yamamoto T, et al. Primary face motor area as the motor representation of articulation. J Neurol 2007;254:442-447.

33. Kim JS, Kwon SU, Lee TG. Pure dysarthria due to small cortical stroke. Neurology 2003;60:1178-1180.

34. Kraus MF, Susmaras T, Caughlin BP, Walker CJ, Sweeney JA, Little DM. White matter integrity and cognition in chronic traumatic brain injury: a diffusion tensor imaging study. Brain 2007;130(pt 10):2508-2519.

35. Ewing-Cobbs L, Prasad MR, Swank P, et al. Arrested development and disrupted callosal microstructure following pediatric traumatic brain injury: relation to neurobehavioral outcomes. Neuroimage 2008;42:1305-1315. 


\section{Neurology}

\section{Corticobulbar tract changes as predictors of dysarthria in childhood brain injury}

Frédérique Liégeois, Jacques-Donald Tournier, Lauren Pigdon, et al.

Neurology 2013;80;926-932 Published Online before print February 6, 2013

DOI 10.1212/WNL.0b013e3182840c6d

This information is current as of February 6, 2013

\begin{tabular}{|c|c|}
\hline $\begin{array}{l}\text { Updated Information \& } \\
\text { Services }\end{array}$ & $\begin{array}{l}\text { including high resolution figures, can be found at: } \\
\text { http://n.neurology.org/content/80/10/926.full }\end{array}$ \\
\hline Supplementary Material & $\begin{array}{l}\text { Supplementary material can be found at: } \\
\text { http://n.neurology.org/content/supp1/2013/02/07/WNL.0b013e } 3182840 \\
\text { c6d.DC1 }\end{array}$ \\
\hline References & $\begin{array}{l}\text { This article cites } 33 \text { articles, } 4 \text { of which you can access for free at: } \\
\text { http://n.neurology.org/content/80/10/926.full\#ref-list-1 }\end{array}$ \\
\hline Subspecialty Collections & $\begin{array}{l}\text { This article, along with others on similar topics, appears in the } \\
\text { following collection(s): } \\
\text { Brain trauma } \\
\text { http://n.neurology.org/cgi/collection/brain_trauma } \\
\text { Developmental disorders } \\
\text { http://n.neurology.org/cgi/collection/developmental_disorders } \\
\text { DWI } \\
\text { http://n.neurology.org/cgi/collection/dwi } \\
\text { MRI } \\
\text { http://n.neurology.org/cgi/collection/mri }\end{array}$ \\
\hline Permissions \& Licensing & $\begin{array}{l}\text { Information about reproducing this article in parts (figures,tables) or in } \\
\text { its entirety can be found online at: } \\
\text { http://www.neurology.org/about/about_the_journal\#permissions }\end{array}$ \\
\hline Reprints & $\begin{array}{l}\text { Information about ordering reprints can be found online: } \\
\text { http://n.neurology.org/subscribers/advertise }\end{array}$ \\
\hline
\end{tabular}

Neurology ${ }^{\circledR}$ is the official journal of the American Academy of Neurology. Published continuously since 1951, it is now a weekly with 48 issues per year. Copyright @ 2013 American Academy of Neurology. All rights reserved. Print ISSN: 0028-3878. Online ISSN: 1526-632X.

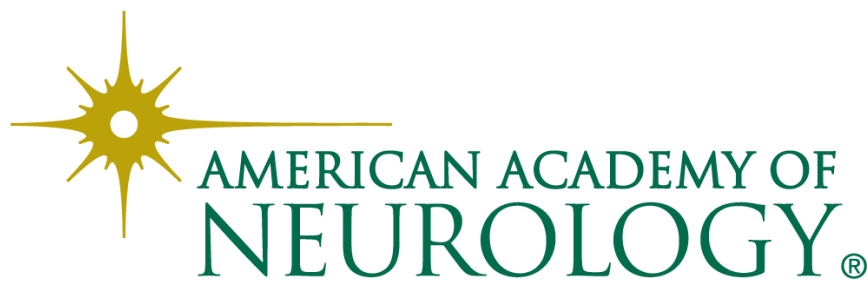

